\title{
Infinite Regular Hexagon Sequences on a Triangle
}

Alvy Ray Smith

\section{CONTENTS}

Introduction

1. Hex Operators

2. Fukuta's Problems

3. Terminology

4. Sameness

5. Eigenpolygon Analysis

6. Algebraic Structure

7. Infinite Regular Hexagon Sequences

8. Deep Structure

References
The well-known dual pair of Napoleon equilateral triangles intrinsic to each triangle is extended to infinite sequences of them, shown to be special cases of infinite regular hexagon sequences on each triangle. A set of hexagon-to-hexagon transformations, the hex operators, is defined for this purpose, a set forming an abelian monoid under function composition. The sequences result from arbitrary strings of hex operators applied to a particular truncation of a given triangle to a hexagon. The deep structure of the sequence constructions reveals surprising infinite sequences of nonconcentric, symmetric equilateral triangle pairs parallel to one of the sequences of hexagons and provides the most visually striking contribution. Extensive experimentation with a plane geometry educational program inspired all theorems, proofs of which utilize eigenvector analysis of polygons in the complex plane.

\section{INTRODUCTION}

This paper uses the "eigenpolygon" decomposition of polygons in the complex plane to extend the wellknown pair of Napoleon equilateral triangles intrinsic to each triangle to infinite sequences of such triangles. These sequences, in turn, are special cases of infinite sequences of regular hexagons on each triangle.

Another theme is the benefit of experimental use of computer graphics in plane geometry. The geometric constructions in this study are tediousoften infeasible - for the unaided person, yet the intuitions gained from dynamic interaction with the complicated constructions are powerful. Each theorem in this paper is the direct result of conjecture inspired by experimentation with normally unwieldy geometric constructions, made possible by the educational software [Sketchpad n.d.; Bennett 1992].

Napoleon's Theorem describes a transformation mapping an arbitrary triangle to an equilateral triangle [Chang and Sederberg 1997, pp. 57-61, 90-98; Coxeter and Greitzer 1967, 60-65; Wetzel 1992]. It is actually a dual pair of transformations leading 
to the so-called outward and inward Napoleon triangles, called positive and negative here for consistency. Fukuta generalizes the Napoleon transformation to a 2-step transformation that converts an arbitrary triangle to a regular hexagon [Fukuta 1996b; Lossers 1997] (see also [Garfunkel and Stahl 1965]) and then to a 3-step transformation yielding a different regular hexagon [Fukuta 1996a; Chapman 1997] strongly concentric with the first, meaning they are parallel as well (Figure 1). Each transformation is parameterized by $\sigma$. At $\sigma=0$, the first Fukuta hexagon is the positive Napoleon triangle plus its Starof-David complementary equilateral. Similarly, all hexagon sequences in the paper can be interpreted as equilateral triangle sequences. At $\sigma=0$, most include one or both Napoleon triangles.

We show that iteration of the middle step in the 3 -step Fukuta transformation creates an infinite sequence of strongly concentric regular hexagons, each being 2 times (the size of) its predecessor (in edge length). The set of two transformations is enlarged to an infinite set by generalizing to what are called the hexagon construction operators, or hex operators, and applying them iteratively or in any order to generate infinite sequences of concentric regular hexagons. One such sequence has each hexagon $\sqrt{3}$ times its predecessor and rotated $\pi / 6$ from it (see Figure 2). Another has each hexagon 2 or 3 times a preceding one and strongly concentric with it (see Figure 3 ). The structure of the transformation set itself is shown to be an abelian monoid in the case of interest.

Moreover, the deep structure of the hexagon sequences reveals surprising infinite sequences of nonconcentric, symmetric equilateral triangle pairs parallel to one of the sequences of hexagons. Each emerges from a chaos of irregular and regular hexagons (Figure 4) in the most visually interesting contribution of the paper (Figure 5).

\section{HEX OPERATORS}

An arbitrary hexagon of six points $H_{1} H_{2} H_{3} H_{4} H_{5} H_{6}$ is abbreviated $H^{*}$, with $H_{i}$ an arbitrary vertex. All arithmetic on subscripts $i$ is modulo 6. Pairs $H_{i} H_{i+3}$ are the main diagonals. $0^{*}$ is the degenerate hexagon at the origin. A positive (negative) triangle has vertices in counterclockwise (clockwise) order.
Define the positive $n$-interlaced hex operator $\mathbf{I}_{n}$ on hexagon $H^{*}$ : For $n \geq 0$ and all $i$, erect positive equilateral triangle $R_{i} H_{i+1} H_{i-n}$, a generating triangle. Then $\mathbf{I}_{n} H^{*}$ is hexagon $R^{*}$. The negative $n$-interlaced hex operator $\mathbf{i}_{n}$ is defined similarly but with negative generating triangles $r_{i} H_{i+1} H_{i-n}$.

Mnemonic names are assigned for $n \leq 2$. $\mathbf{P}:=$ $\mathbf{I}_{0}$ and $\mathbf{p}:=\mathbf{i}_{0}$ are the progressive hex operators, since each builds equilaterals on successive pairs of vertices. The nonprogressive ones are $\mathbf{I}:=\mathbf{I}_{1}$ and $\mathbf{i}:=\mathbf{i}_{1}$, the interlaced hex operators, and $\mathbf{B}:=\mathbf{I}_{2}$ and $\mathbf{b}:=\mathbf{i}_{2}$, which are bi-interlaced.

Let $\mathbf{F}$ be the set of hex operators and $\mathbf{F}^{+}$the set of nonempty compositions on $\mathbf{F}$. These are written as concatenations - for example, IP $P^{*}$ means $\mathbf{I}\left(\mathbf{P}\left(P^{*}\right)\right)$. Letting the empty string $\varphi$ represent the identity, mapping a hexagon to itself, $\mathbf{F}^{*}=\mathbf{F}^{+} \cup\{\varphi\}$ is the set of strings of hex operators. The principal purpose here is to generalize the Fukuta (Napoleon) results to infinite sequences of hexagons (equilaterals) on a triangle by exploring the actions of arbitrary strings in $\mathbf{F}^{*}$.

Define the hex operator iterate by example: $\mathbf{P}^{n}$, for $n \geq 0$, is defined by $\mathbf{P}^{0}=\varphi, \mathbf{P}^{1}=\mathbf{P}, \mathbf{P}^{n+1}=$ $\mathbf{P P}^{n}$.

Define the successive centroids operator $\mathbf{C}$ on a hexagon $H^{*}$ : Find the centroid $C_{i}$ of each successive triplet $H_{i-1} H_{i} H_{i+1}$ of vertices of $H^{*}$. Then $\mathbf{C} H^{*}$ is the hexagon $C^{*}$.

\section{FUKUTA'S PROBLEMS}

In an arbitrary triangle $A B C$, let $\left(P_{1}, P_{2}\right),\left(P_{3}, P_{4}\right)$, and $\left(P_{5}, P_{6}\right)$ be the pairs of points on the sides $B C$, $C A$, and $A B$ respectively, such that $A P_{5} P_{4}, P_{6} B P_{1}$, and $P_{3} P_{2} C$ are congruent with one another and similar to $A B C$-i.e., $P^{*}$ is the hexagon obtained by truncating a copy of $A P_{5} P_{4}$ from each vertex of $A B C$. (See Figure 1.) Hence $P^{*}$ is called a truncation of $A B C$, parameterized by $\sigma=\left|B P_{1}\right| /|B C|$, with $0 \leq \sigma \leq 1$. If $\bar{\sigma}:=1-\sigma$, then $P_{1}=\sigma C+\bar{\sigma} B$, and similarly for all $P_{i}$. Let $A^{\prime}, B^{\prime}, C^{\prime}$ be the points of intersection of $P_{1} P_{4}$ and $P_{2} P_{5}, P_{3} P_{6}$ and $P_{1} P_{4}$, and $P_{2} P_{5}$ and $P_{3} P_{6}$, respectively.

Figure 1 shows the results of applying $\mathbf{P}$ and $\mathbf{I P}$ to the truncation $P^{*}$ of triangle $A B C: R^{*}=\mathbf{P} P^{*}$ and IP $P^{*}$ (dashed) are irregular hexagons. Remarkably, both $G_{0}^{*}=\mathbf{C P} P^{*}$ and $G_{1}^{*}=\mathbf{C I P} P^{*}$ (solid bold) 


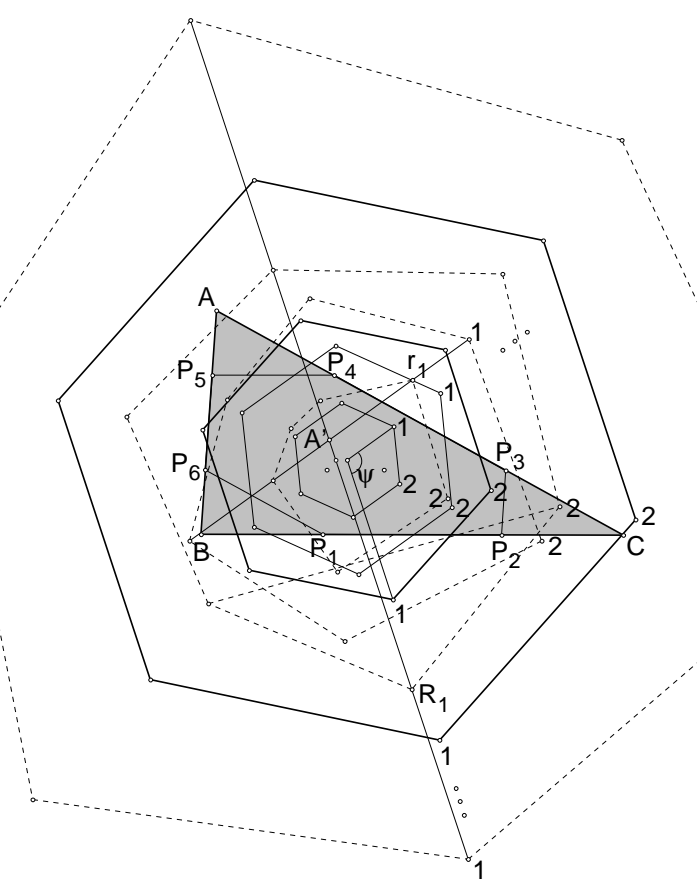

FIGURE 1. First two elements of sequences $\boldsymbol{G}$ (bold) and $\boldsymbol{g}$ (light), hence $\boldsymbol{S}$ and $\boldsymbol{s}$.

are regular hexagons concentric with $A B C$ - that is, centered on its centroid. The two hexagons are strongly concentric (parallel and concentric), and one of them is 2 times the other, and similarly for $g_{0}^{*}=\mathbf{C} \mathbf{p} P^{*}$ and $g_{1}^{*}=\mathbf{C i p} P^{*}$ (solid light). They are superimposed in Figure 1 to show that the two sets of regular hexagons are different, in general, and not strongly concentric with one another. Let $\psi$ denote the angle between positive and negative cases.

Many other interesting aspects of Fukuta's problems are indicated: The main diagonals of $\mathbf{I P} P^{*}$ are concurrent, equal in length, equally spaced radially, parallel to the sides of $\mathbf{C I P} P^{*}$ and 3 times their size. So are the main diagonals of $\mathbf{P} P^{*}$ (relative to $\mathbf{C P} P^{*}$ ), which are a subset of the main diagonals of IP $P^{*}$. Similar results hold for the main diagonals of $\mathbf{i p} P^{*}$ and $\mathbf{p} P^{*}$. Furthermore, they intersect the main diagonals of $\mathbf{I P} P^{*}$ and $\mathbf{P} P^{*}$ at the points $A^{\prime}, B^{\prime}$, and $C^{\prime}$. The regular hexagons $\mathbf{C P} P^{*}$ and $\mathbf{C p} P^{*}$ are strongly concentric if $A B C$ is isosceles, as are $\mathbf{C I P} P^{*}$ and $\operatorname{Cip} P^{*}$. In this case, $\psi=0$; generally $\psi$ varies with $A B C$ but is independent of $\sigma$. $\mathbf{C P} P^{*}$ and $\mathbf{C p} P^{*}$ become the same hexagon in the degenerate case of an isosceles triangle with height 0 , its base bisected by the third vertex. These other aspects also generalize but, for brevity, will not be further pursued.

\section{TERMINOLOGY}

For orientation, only the first and second vertex of a hexagon are labeled, generally by 1 and 2 . A single shaded triangle $A B C$ is used in all figures (to within scaling) for comparison, so the redundant labels $A$, $B, C$, and $P_{i}$ are omitted in figures after Figure 1, as are the main diagonals and triangle $A^{\prime} B^{\prime} C^{\prime}$.

All sequences here begin with a construction, the initialization, on truncation $P^{*}$ of $A B C$, which is normally progressive, though nonprogressive initializations are also treated. A typical procedure is: (1) Truncate a triangle to a hexagon. (2) Apply a hex operator to the result of the preceding step. (3) Repeat step 2 a number $j \geq 0$ of times with various hex operators. (4) Apply $\mathbf{C}$ to the hexagon from step 3 to yield sequence member $H_{j}^{*}$. Step 2 for $j=0$ is the initialization.

A hex operator applied to a hexagon yields a generating hexagon. Use of successive centroids operator $\mathbf{C}$ on a generating hexagon is a reduction of it. In Figure 1, $\mathbf{P} P^{*}$ is the irregular generating hexagon on truncation $P^{*}$, and $\mathbf{C P} P^{*}$ is the reduction of it to a regular one. In all figures generating hexagons are dashed and reductions of them solid.

It is useful to embed constructions in the complex plane, with origin at the centroid of $A B C$, so $A+B+$ $C=0$. Thus $P_{i}+P_{i+2}+P_{i+4}=0$ by expanding each in terms of $\sigma$. The centroid of an arbitrary triangle $P Q R$ is $\frac{1}{3}(P+Q+R)$. Define operators $\omega=e^{i \pi / 3}$ and $\tau=e^{i \pi / 6}$, with conjugates $\bar{\omega}$ and $\bar{\tau}$. A positive regular hexagon $H^{*}$ centered on the origin, vertices increasing counterclockwise, satisfies $H_{i+1}=\omega H_{i}$, and a negative regular one $H_{i+1}=\bar{\omega} H_{i}$. A positive equilateral triangle - for example, $R_{1} P_{2} P_{1}$ in Figure 1 - is described by $R_{1}=\bar{\omega} P_{2}+\omega P_{1}$; a negative one by $r_{1}=\omega P_{2}+\bar{\omega} P_{1}$.

\section{SAMENESS}

Define an equivalence relation $\cong$, called sameness, by the condition that $G^{*} \cong H^{*}$ if hexagon $G^{*}$ is congruent, without rotation or translation, to hexagon $H^{*}$. Thus two concentric hexagons are the same if they are identical when vertex order and labels are ignored.

Consider a hexagon $H^{*}$ (from a given class of hexagons) and hex operator strings $\mathbf{F}_{1}, \mathbf{F}_{2} \in \mathbf{F}^{*}$. If $\mathbf{C F}_{1} H^{*} \cong \mathbf{C F}_{2} H^{*}$, then write $\mathbf{F}_{1} \cong \mathbf{F}_{2}$ (for that 
class). If $\mathbf{C F}_{1} H^{*} \cong s \mathbf{C F}_{2} H^{*}$, where $s$ is an arbitrary scalar constant, write $\mathbf{F}_{1} \cong s \mathbf{F}_{2}$. Similarly, if $\mathbf{C F}_{1} H^{*} \cong e^{i \theta} \mathbf{C F}_{2} H^{*}$, write $\mathbf{F}_{1} \cong e^{i \theta} \mathbf{F}_{2}$. For regular hexagons write $\mathbf{F}_{1} \cong e^{i \theta \bmod (\pi / 3)} \mathbf{F}_{2}$ - for example, $\theta= \pm \pi / 2$ implies $\mathbf{F}_{1} \cong \tau \mathbf{F}_{2}$, while $\theta= \pm n \pi / 3$, for $n$ integer, implies $\mathbf{F}_{1} \cong \mathbf{F}_{2}$.

As a first application of sameness, consider the $n$-interlaced hex operators. It is not difficult to see that the positive (negative) 3 - and 4 -interlaced hex operators are just $\mathbf{i}$ and $\mathbf{p}(\mathbf{I}$ and $\mathbf{P})$, respectively, using $\cong$ equivalence. Thus it suffices to restrict attention to $n \leq 2$. Since $\mathbf{B}$ and $\mathbf{b}$ are the same, only $\mathbf{B}$ is studied. Hence sameness collapses the set of hex operators to $\mathbf{F}=\{\mathbf{P}, \mathbf{I}, \mathbf{B}, \mathbf{p}, \mathbf{i}\}$.

\section{EIGENPOLYGON ANALYSIS}

The (backward) shift operator $\mathbf{S}$ is defined for hexagon $G^{*}$ by $H^{*}=\mathbf{S} G^{*}$ with $H_{i}=G_{i+1}$, and its inverse $\overline{\mathbf{S}}$ by $H^{*}=\overline{\mathbf{S}} G^{*}$ with $H_{i}=G_{i-1}$. As shown in [Chang and Sederberg 1997], $\mathbf{S}$ is a linear operator with eigenvalues $\omega^{i}$, the sixth roots of unity, and

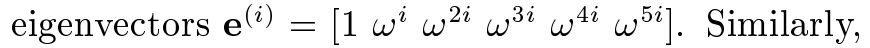
$\overline{\mathbf{S}}$ is a linear operator with the same eigenvectors but with eigenvalues $\bar{\omega}^{i}$. Any hexagon can be expressed as a complex linear sum of these eigenvectors - hence "eigenpolygons" or "basis polygons" [Glassner 1999]. For a polygon with centroid at the origin, as assumed here, the sixth eigenvector is not used.

All hex operators can be written as expressions of the identity and shift operators: $\mathbf{I}_{n}=\bar{\omega} \mathbf{S}+\omega \overline{\mathbf{S}}^{n}$ and $\mathbf{i}_{n}=\omega \mathbf{S}+\bar{\omega} \overline{\mathbf{S}}^{n}$. So can the successive centroids operator: $\mathbf{C}=\frac{1}{3}(\mathbf{S}+\varphi+\overline{\mathbf{S}})$. Thus all operators of interest are linear, with the eigenvectors above and easily computed eigenvalues: $\omega^{i-1}+\bar{\omega}^{n i-1}$ and $\omega^{i+1}+$ $\bar{\omega}^{n i+1}$ for the hex operators, respectively, and $\frac{1}{3}\left(\omega^{i}+\right.$ $\left.1+\bar{\omega}^{i}\right)$ for $\mathbf{C}$. Let $\lambda_{\mathbf{X}}$ be the vector of eigenvalues for operator $\mathbf{X}$. Then, with simplification,

$$
\begin{aligned}
& \lambda_{\mathbf{P}}=\left[\begin{array}{llllll}
\omega+1 & 2 \omega & \omega(\omega+1) & \omega^{2} & 0 & 1
\end{array}\right], \\
& \lambda_{\mathbf{p}}=\left[\begin{array}{lllll}
0 & -\omega & -\omega(\omega+1) & -2 \omega^{2}-\omega^{2}(\omega+1) & 1
\end{array}\right], \\
& \lambda_{\mathbf{I}}=\left[\begin{array}{llllll}
2 & 1 & -1 & -2 & -1 & 1
\end{array}\right], \\
& \lambda_{\mathbf{i}}=\left[\begin{array}{llllll}
-1 & -2 & -1 & 1 & 2 & 1
\end{array}\right], \\
& \lambda_{\mathbf{B}}=\left[\begin{array}{lllll}
-\omega^{2}(\omega+1) & \omega^{2} \omega(\omega+1) & -\omega & -(\omega+1) & 1
\end{array}\right], \\
& \lambda_{\mathbf{C}}=\left[\begin{array}{llllll}
\frac{2}{3} & 0 & -\frac{1}{3} & 0 & \frac{2}{3} & 1
\end{array}\right] .
\end{aligned}
$$

Let $\lambda_{\mathbf{X Y}}$ be the vector obtained from pairwise multiplication of $\lambda_{\mathbf{X}}$ and $\lambda_{\mathbf{Y}}$ : for example,

$$
\lambda_{\mathbf{C P}}=\left[\frac{2}{3}(\omega+1) \quad 0-\frac{1}{3} \omega(\omega+1) \quad 0 \quad 001\right] .
$$

The eigenpolygon decomposition of truncation $P^{*}$ has particular importance here. Let $E$ be the $6 \times 6$ matrix where each row $i$ is eigenvector $\mathbf{e}^{(i)}$ and each column $i$ is called $\mathbf{E}^{(i)}$. Then $P^{*}=\mathbf{a} E$ for complex coefficients a. Inverting $E$ yields a in terms of given parameters:

$\mathbf{a}=\frac{1}{6}\left[\begin{array}{llllll}-\bar{\sigma} \omega^{2}(\omega+1) V & \rho \omega^{2} v & 0 & -\rho \omega V & \bar{\sigma}(\omega+1) v & 0\end{array}\right]$, where $V=-A+\omega B+\bar{\omega} C, v=-A+\bar{\omega} B+\omega C$, and $\rho=1-3 \sigma$. Then the effect of operator $\mathbf{X}$ on truncation $P^{*}$ is computed from $\mathbf{X} P^{*}=\sum\left(\lambda_{\mathbf{X}}\right)_{i} \mathbf{a}_{i} \mathbf{e}^{(i)}=$ $\sum \mathbf{a}_{\mathbf{x}_{i}} \mathbf{e}^{(i)}=\mathbf{a} \mathbf{x} \cdot \mathbf{E}^{(i)}$, where $\mathbf{a}_{\mathbf{X}}$ is the vector obtained from pairwise multiplication of $\lambda_{\mathbf{X}}$ and $\mathbf{a}$. All elements of $\mathbf{a}_{\mathbf{C P}}$ are 0 except the first, so $\mathbf{C P} P^{*}$ must be a regular hexagon, the first eigenpolygon $\mathbf{e}^{(1)}$, with size and orientation given by multiplier $(\bar{\sigma} / 3) V$ - that is, $G_{0}^{*}=(\bar{\sigma} / 3) V \mathbf{e}^{(1)}$. This is just the solution to Fukuta's first problem. Similar analysis for $\operatorname{CIP} P^{*}$ shows the same result but with additional multiplier $\left(\lambda_{\mathbf{I}}\right)_{1}=2$-i.e., $G_{1}^{*}=2(\bar{\sigma} / 3) V \mathbf{e}^{(1)}$, the solution to Fukuta's second problem. The operators annihilate all but one eigenpolygon, which is a regular hexagon. Similarly for the negative Fukuta cases: $g_{0}^{*}=(\bar{\sigma} / 3) v \mathbf{e}^{(5)}, g_{1}^{*}=2(\bar{\sigma} / 3) v \mathbf{e}^{(5)}$. At $\sigma=0$, the hexagons $G_{0}^{*}=\frac{1}{3} V \mathbf{e}^{(1)} g_{0}^{*}=\frac{1}{3} v \mathbf{e}^{(5)}$, the Napoleon hexagons, include the Napoleon equilaterals.

\section{ALGEBRAIC STRUCTURE}

The algebraic structure of $\mathbf{F}$ is established first for hex operators applied to arbitrary hexagons, then refined for application to hexagons that are regular under reduction by $\mathbf{C}$. The following useful result can be established easily:

Lemma 1 (Duality). Let $H^{*}$ be an arbitrary hexagon and $h^{*}$ the same hexagon with vertices ordered oppositely.

(i) $\mathbf{P} h^{*} \cong \mathbf{p} H^{*}$.

(ii) $\mathbf{p} h^{*} \cong \mathbf{P} H^{*}$.

(iii) $\mathbf{I} h^{*} \cong \mathbf{i} H^{*}$.

(iv) $\mathbf{i} h^{*} \cong \mathbf{I} H^{*}$.

(v) $\mathbf{B} h^{*} \cong \mathbf{B} H^{*}$. 
So $\mathbf{P}$ and $\mathbf{p}$ are duals on vertex order, as are $\mathbf{I}$ and $\mathbf{i}$; and $\mathbf{B}$ is self-dual. Thus it suffices to state and prove a theorem for the positive case only.

It is easily checked that all hex operators are commutative and associative, hence:

Lemma 2 (Semigroup). The hex operators $\mathbf{F}$ under function composition form an abelian semigroup.

There is now enough machinery to derive the principal tool for special hexagons:

Lemma 3 (Identity). If $\mathbf{C} H^{*}$ is positive regular, then so are $\mathbf{C P} H^{*}, \mathbf{C I} H^{*}, \mathbf{C B} H^{*} \cong \mathbf{C P} H^{*}, \mathbf{C p} H^{*}$, and $\mathbf{C i} H^{*}$, with $\mathbf{P} \cong \sqrt{3} \tau$ (and $\left.\mathbf{P}^{2} \cong 3\right), \mathbf{I} \cong 2, \mathbf{B} \cong \mathbf{P}$, $\mathbf{p} \cong 0$, and $\mathbf{i} \cong 1$.

Proof. Saying that $\mathbf{C} H^{*}$ is positive regular amounts to the requirement that all eigenpolygons in its decomposition be annihilated but the first one. Then clearly $\mathbf{I C} H^{*}$ simply multiplies the given hexagon by 2 - that is, the only operative eigenvalue in $\lambda_{\mathbf{I}}$ is $2-$ for $\mathbf{I C H} H^{*}=2 \mathbf{C} H^{*}$. But $\mathbf{I}$ and $\mathbf{C}$ commute, so $\mathbf{I C} H^{*}=\mathbf{C I} H^{*}$. The other results follow from operative eigenvalues $\omega+1=\sqrt{3} \tau, \bar{\omega}+1=\sqrt{3} \bar{\tau}$, 0 , and -1 , respectively.

The negative case follows by duality: $\mathbf{P} \cong 0, \mathbf{I} \cong 1$, $\mathbf{B} \cong \mathbf{p}, \mathbf{p} \cong \sqrt{3} \tau$ (and $\mathbf{p}^{2} \cong 3$ ), and $\mathbf{i} \cong 2$ for $\mathbf{C} H^{*}$ negative regular.

The next result is an immediate consequence of the fact that $\mathbf{i}$ is the unique identity for the Lemma 2 semigroup in a special case:

Lemma 4 (Monoid). The hex operators $\mathbf{F}$, restricted to hexagons that reduce under $\mathbf{C}$ to positive regular, form an abelian monoid under function composition.

So, in the monoid, we have $\left(\mathbf{F}_{1} \mathbf{F}_{2}\right) \mathbf{F}_{3} \cong \mathbf{F}_{1}\left(\mathbf{F}_{2} \mathbf{F}_{3}\right)$, $\mathbf{F}_{1} \mathbf{F}_{2} \cong \mathbf{F}_{2} \mathbf{F}_{1}$, and $\mathbf{i} \mathbf{F}_{1} \cong \mathbf{F}_{1} \mathbf{i} \cong \mathbf{F}_{1}$, for $\mathbf{F}_{1}, \mathbf{F}_{2}$, $\mathbf{F}_{3} \in \mathbf{F}^{*}$. Because $\mathbf{B} \cong \mathbf{P}, \mathbf{B}$ is redundant in the monoid.

The first Fukuta transformation gives $\mathbf{C P} P^{*}$ positive regular, so iteration of Lemma 3 yields:

Lemma 5 (Progressive Initialization). For $\mathbf{F}_{1} \mathbf{P} P^{*}$ a hexagon construction on the truncation $P^{*}$ of $A B C$, with $\mathbf{F}_{1} \in \mathbf{F}^{*}$, reduction by $\mathbf{C}$ yields a positive regular hexagon; that is, $\mathbf{C F}_{1} \mathbf{P} P^{*}$ is positive regular.

Figure 2 shows the result of applying $\mathbf{P}$ or $\mathbf{p}$ iteratively to truncation $P^{*}$.

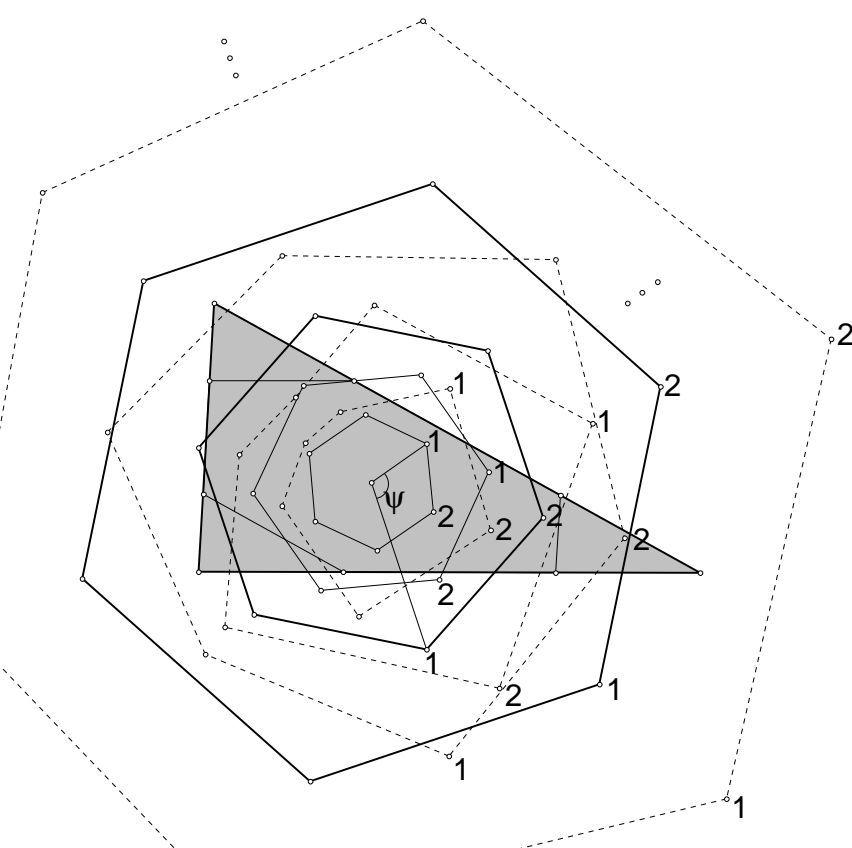

FIGURE 2. First two elements in sequences $\boldsymbol{H}$ (bold) and $\boldsymbol{h}$ (light).

Eigenpolygon analysis shows that irregular hexagons result from initialization by $\mathbf{I}, \mathbf{B}$, or $\mathbf{i}$ instead of $\mathbf{P}$ or $\mathbf{p}$. The following result complements Lemma 5 by stating when a construction on a nonprogressive initialization may too yield a regular hexagon.

Lemma 6 (Nonprogressive initialization). For $\mathbf{F}_{1} \mathbf{I} P^{*}$ on truncation $P^{*}$, with $\mathbf{F}_{1} \in \mathbf{F}^{*}$, reduction by $\mathbf{C P}$ (instead of $\mathbf{C})$ yields a positive regular hexagon; that is, $\mathbf{C P F}_{1} \mathbf{I} P^{*}$ is positive regular. The same is true if $\mathbf{I}$ is replaced by $\mathbf{B}$.

Proof. By commutativity, $\mathbf{C P F}_{1} \mathbf{I} P^{*}=\mathbf{C F}_{1} \mathbf{I P} P^{*}$, which is positive regular by Lemma 5 . Similarly for B.

Hexagon sequences generated by strings $\mathbf{F}_{1}$ containing no operators $\mathbf{P}$ or $\mathbf{p}$ can be shown irregular by eigenpolygon analysis, so Lemma 6 states that no new regular hexagons result from nonprogressive initializations. It also implies that each regular hexagon sequence can be generated in a different way: for example, in place of construction $\mathbf{I}^{a} \mathbf{P}^{b} \mathbf{P} P^{*}$ reduced by $\mathbf{C}$, use construction $\mathbf{I}^{a} \mathbf{B}^{b} P^{*}$ reduced by $\mathbf{C P}$ to the same regular hexagon. $\mathbf{B} \cong \mathbf{P}$ in this case only after an application of at least one $\mathbf{P}$; hence $\mathbf{B}$ is 
not redundant in $\mathbf{F}$, in general, although it is in the monoid.

\section{INFINITE REGULAR HEXAGON SEQUENCES}

The most general regular hexagon sequences due to the hex operators are described next, where $H^{*}$ a 2 3 multiple of $G^{*}$ means $H^{*} \cong 2^{m} 3^{n} G^{*}$, with $m, n \geq$ 0 , and where disjoint means disjoint as sets not as geometry.

Theorem 1. For an arbitrary triangle $A B C$ with truncation $P^{*}$, there exist these infinite, concentric but disjoint, positive regular hexagon sequences centered on $A B C$, generated by strings in $\mathbf{F}^{*}$ for $j \geq 0$ :

$$
\begin{aligned}
\boldsymbol{S} & =\left\{S_{j}^{*}: S_{0}^{*}=\mathbf{C P} P^{*}, S_{j}^{*} \text { a } 2-3 \text { multiple of } S_{0}^{*}=G_{0}^{*}\right\} \\
\boldsymbol{S}_{\tau} & =\left\{S_{\tau_{j}}^{*}: S_{\tau_{0}}^{*}=\mathbf{C P}^{2} P^{*}, S_{\tau_{j}}^{*} \text { a } 2-3 \text { multiple of } S_{\tau_{0}}^{*}=H_{1}^{*}\right\}
\end{aligned}
$$

Proof. By Lemma 3, iterates of $\mathbf{I}$ and $\mathbf{P}^{2}$ multiply a positive regular hexagon by powers of 2 or 3 , respectively, into strongly concentric positive regular hexagons of larger size. 2-3 multiples can be ordered uniquely by numeric size. Suppose $2^{m} 3^{n}$ is the next factor in succession. Then $S_{j}^{*}=\mathbf{C I}^{m}\left(\mathbf{P}^{2}\right)^{n} \mathbf{P} P^{*}$ generates the corresponding element of $\boldsymbol{S}$ and is positive regular. $\boldsymbol{S}_{\tau}$ is generated the same way as $\boldsymbol{S}$ but with one extra application of $\mathbf{P}$, which rotates the sequence $\pi / 6$ from $\boldsymbol{S}$. That is, $S_{\tau_{j}}^{*}=$ $\mathbf{C I}^{m}\left(\mathbf{P}^{2}\right)^{n} \mathbf{P}^{2} P^{*} \cong \sqrt{3} \tau S_{j}^{*}$. Each of $\boldsymbol{S}$ and $\boldsymbol{S}_{\tau}$ is strongly concentric.

Figure 3 shows the first seven elements of $\boldsymbol{S}$ and five of $\boldsymbol{S}_{\tau}$. The $\boldsymbol{S}$ elements are obtained by applying $\mathbf{I}^{0}, \mathbf{I}^{1}, \mathbf{P}^{2}, \mathbf{I}^{2}, \mathbf{I}^{1} \mathbf{P}^{2}$ (or its commuted equivalents), $\mathbf{I}^{3}$, and $\mathbf{P}^{4}$, respectively, to $\mathbf{P} P^{*}$. Let $\boldsymbol{s}$ and $\boldsymbol{s}_{\tau}$ be the duals to $\boldsymbol{S}$ and $\boldsymbol{S}_{\tau}$. $\boldsymbol{S}_{\tau}$ and $\boldsymbol{s}_{\tau}$ do not contain Napoleon hexagons at $\sigma=0$.

Theorem 2. The concentric sequence $\boldsymbol{A}=\{\boldsymbol{S}\} \cup$ $\{\boldsymbol{s}\} \cup\left\{\boldsymbol{S}_{\tau}\right\} \cup\left\{\boldsymbol{s}_{\tau}\right\} \cup\left\{0^{*}\right\}$ is a disjoint union, in general, containing every unique regular hexagon (by $\cong$ equivalence) generable by strings in $\mathbf{F}^{*}$.

Proof. Let $\boldsymbol{A}$ be the ordered union of the two sequences of Theorem 1, the two from its dual, and the one element $A_{0}^{*}=0^{*}$ absent from all of them. These are disjoint sets, except in the degenerate case of $A B C$ an isosceles triangle of height 0 , mentioned earlier, when $\boldsymbol{S}$ and $\boldsymbol{s}\left(\boldsymbol{S}_{\tau}\right.$ and $\left.\boldsymbol{s}_{\tau}\right)$ are the same; but

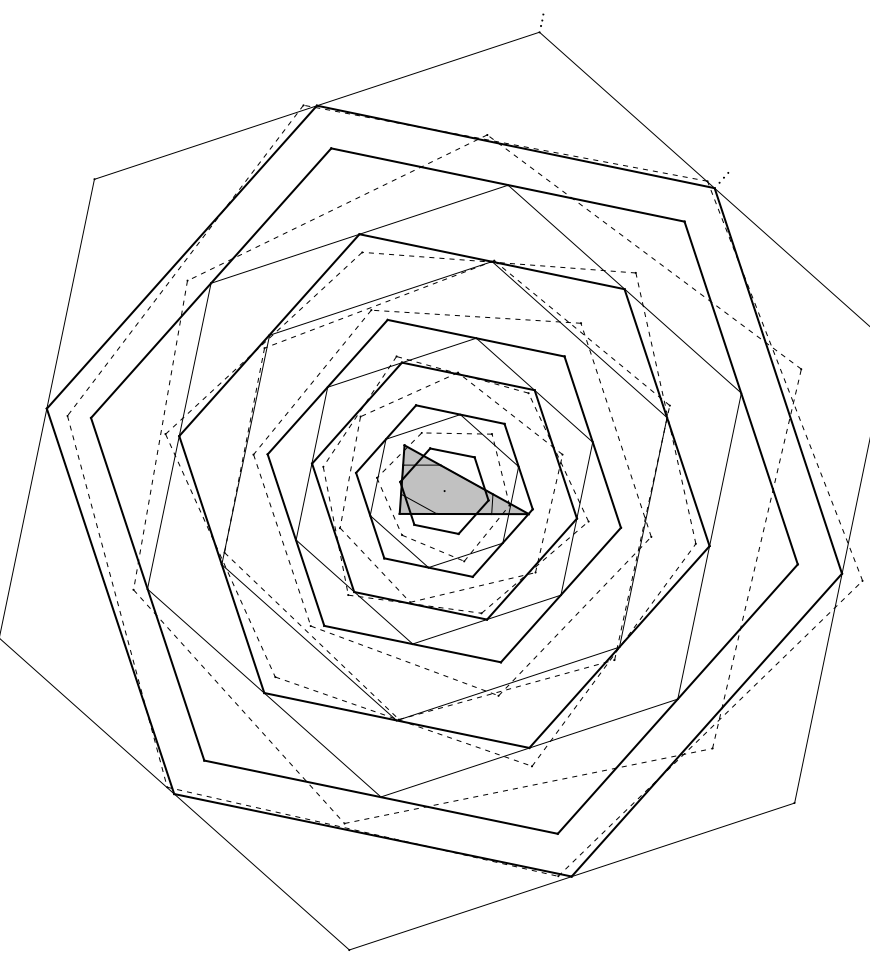

FIGURE 3. First seven elements in sequence $\boldsymbol{S}$ (bold) and first five in $\boldsymbol{S}_{\tau}$ (light).

$\boldsymbol{A}$ is never strongly concentric. Because of commutativity and Lemma 6 (and its dual), it suffices to consider only constructions of the form

\section{$\mathbf{C I} \mathbf{I}^{a} \mathbf{P}^{c} \mathbf{p}^{d} H^{*}$}

on the initializations $H^{*} \in\left\{\mathbf{P} P^{*}, \mathbf{p} P^{*}\right\}$, for nonnegative integers $a, b, c, d$. $\boldsymbol{S}$ and $\boldsymbol{S}_{\tau}$ exhaust all cases for which $c=d=0$ on $H^{*}=\mathbf{P} P^{*}$. For this initialization, $\mathbf{i}^{c}$ with $c>0$, is an identity creating no new hexagons, and $\mathbf{p}^{d}$ with $d>0$ always zeroes to $0^{*}$. The negative case follows by duality, so $\boldsymbol{A}$ exhausts $\mathbf{F}^{*}$.

Simply iterating the elements of $\mathbf{F}$ generates useful infinite regular subsequences of $\boldsymbol{A}$. The following result is easily established by Lemma 3 and simple induction.

Theorem 3. For an arbitrary triangle $A B C$ with truncation $P^{*}$, there exist these infinite concentric positive regular hexagon sequences centered on $A B C$, generated respectively by iterates of $\mathbf{I}$ and $\mathbf{P}$ for $j \geq 0$ :

$$
\begin{aligned}
& \boldsymbol{G}=\left\{G_{j}^{*}: G_{0}^{*}=\mathbf{C P} P^{*}, G_{j+1}^{*} 2 \text { times } G_{j}^{*}\right\} \\
& \boldsymbol{H}=\left\{H_{j}^{*}: H_{0}^{*}=\mathbf{C P} P^{*}, H_{j+1}^{*} \sqrt{3} \text { times } H_{j}^{*} \text { rotated } \frac{\pi}{6}\right\}
\end{aligned}
$$


Let $\boldsymbol{g}$ and $\boldsymbol{h}$ be the dual sequences generated by iterates of $\mathbf{i}$ and $\mathbf{p}$. The first two elements of $\boldsymbol{G}$ and $\boldsymbol{g}$ are shown in Figure 1, and those of $\boldsymbol{H}$ and $\boldsymbol{h}$ in Figure 2. Fukuta's two positive-case hexagons are the first two in sequence $\boldsymbol{G}$, hence $\boldsymbol{S}$; the negativecase hexagons are the first two in $\boldsymbol{g}$, hence $\boldsymbol{s}$. $\boldsymbol{G}$ is strongly concentric and a subset of $\boldsymbol{S}$. The even elements of $\boldsymbol{H}$ are also a subset of $\boldsymbol{S}$, but the odd ones belong to $\boldsymbol{S}_{\tau}$.

\section{DEEP STRUCTURE}

Although identities $\mathbf{i}^{m}$ contribute nothing to hex operator constructions reduced by $\mathbf{C}$, they do induce an interesting regular structure in general. Let $U_{m}^{*}=\mathbf{i}^{m} \mathbf{P} P^{*}$ be the generating hexagon for $\mathbf{i}^{m}$. Figure 4 shows that the identity cluster $\left\{U_{m}^{*}: m \geq 0\right\}$ of distinct generating hexagons all map to one regular hexagon $\mathbf{C P} P^{*}$. Nevertheless, experiments with Sketchpad strongly suggest the sceptre structure defined in the lemma below, where a sceptre (from the acronym of "symmetric, congruent, equilateral, parallel triangles") is always formed by the intersections of two triples of concurrent equiangular lines, with the lines of one triple pairwise parallel those of the other (Figure 4).

Lemma 7 (Identity Cluster). The identity cluster on truncation $P^{*}$ of $A B C$ has these properties:

(i) For each $i$, vertices labeled $i$ (for $m$ even), and $i+3$ (for $m$ odd), form a line $L_{i}$ parallel to a side of hexagons $\boldsymbol{g}$. Call $L_{i}$ a vertex locus.

(ii) At $\sigma=\frac{1}{3}$, every hexagon $U_{m}^{*}$ is the same as the Napoleon hexagon, strongly concentric with its reduction by $\mathbf{C}$, and $\frac{3}{2}$ is its size: $U_{m}^{*} \cong \frac{3}{2} \mathbf{C} U_{m}^{*}$.

(iii) $L_{i}, L_{i+2}, L_{i+4}$ are concurrent. Let $\mathbf{L}_{1}, \mathbf{L}_{2}$ be the triples for $i=1,2$, and let $K_{1}$ and $K_{2}$ be the corresponding points of concurrency.

(iv) The elements of $\mathbf{L}_{2}$ intersect $C, A$, and $B$, respectively.

(v) The intersection points of $\mathbf{L}_{1}$ and $\mathbf{L}_{2}$ form a sceptre, that is, a pair of congruent parallel equilateral triangles $\Delta_{1}=K_{1} T_{3} T_{4}$ and $\Delta_{2}=K_{2} T_{6} T_{1}$, with $T_{i}=L_{i} \cap L_{i+1}$. The sceptre is parallel to the hexagons $\boldsymbol{g}$.

(vi) $K_{1} K_{2}$ is collinear with the centroid of $A B C$. The distance $\left|K_{1} K_{2}\right|$ is the length of the main diagonals of the identity cluster.

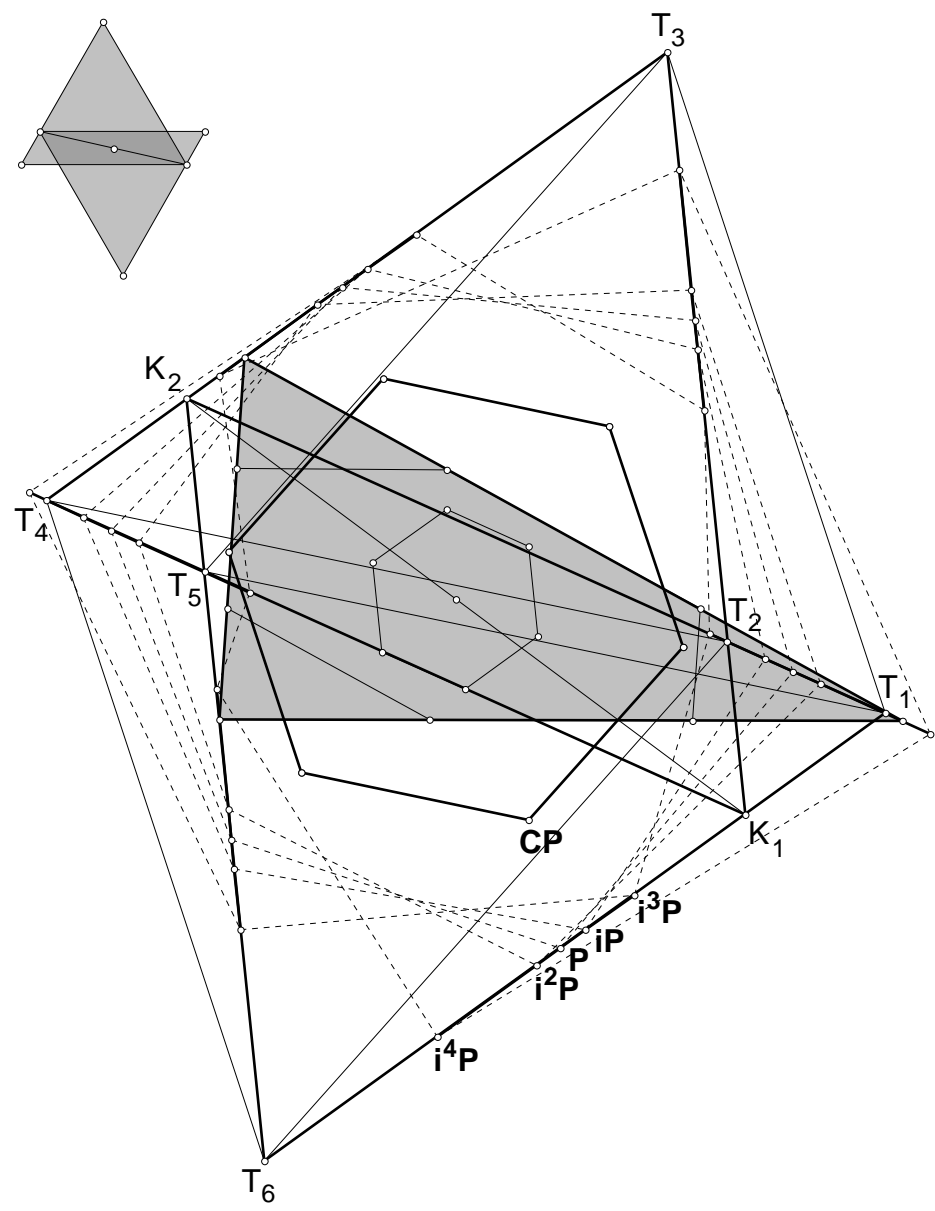

FIGURE 4. Identity cluster generating hexagons near $\sigma=\frac{1}{3}$. Sceptre motif is at upper left.

(vii) At $\sigma=\frac{1}{3}, K_{1}$ and $K_{2}$ are symmetric about the origin. At $\sigma=\frac{2}{3}, K_{1}$ or $K_{2}$ is coincident with the origin. At $\sigma=1, K_{1}$ and $K_{2}$ are coincident. (viii) $T_{6} T_{1} T_{3} T_{4}$ is a parallelogram with angle $\psi$. We have $T_{6} T_{1} \in \Delta_{1}, T_{3} T_{4} \in \Delta_{2}$, and $\left|T_{1} T_{3}\right|=\left|K_{1} K_{2}\right|$. The triangles $T_{1} T_{3} T_{5}$ and $T_{2} T_{4} T_{6}$ are equilateral, congruent and parallel the hexagons $\boldsymbol{G}$, with sides 3 times those of $G_{0}^{*}$.

Proof. (i) $U_{m}^{*}=(-1)^{m} s V \mathbf{e}^{(1)}+(-2)^{m+1} r v \mathbf{e}^{(2)}+r V \mathbf{e}^{(4)}$, with $r=\rho / 6$ and $s=\bar{\sigma} / 2$. Experiment suggests that $U_{m}^{*}-\mathbf{S}^{3} U_{m-1}^{*}$ are vertex loci. Indeed, $U_{m}^{*}-$ $\mathbf{S}^{3} U_{m-1}^{*}=2(-2)^{m-1} \rho v \mathbf{e}^{(2)}$ for $m \geq 1$, and induction on $m$ proves that all these lines must pass through $U_{0}^{*}$. So $L_{i}$ has direction $v \mathbf{e}_{i}^{(2)}$, parallel sides of hexagons $\boldsymbol{g}$.

(ii) $\sigma=\frac{1}{3}$ gives $r=0, s=\frac{1}{3}$, and $U_{m}^{*}=\frac{1}{3}(-1)^{m} V \mathbf{e}^{(1)}$, a regular hexagon the same as the Napoleon hexagon, which reduces to $\mathbf{C} U_{m}^{*}=\frac{2}{9}(-1)^{m} V \mathbf{e}^{(1)} \cong G_{0}^{*}$. 
(iii) Without loss of generality, the equations for $L_{i}$ can be computed from $U_{2}^{*}$ and $U_{0}^{*}$ as equations

$$
\left(\bar{U}_{2}^{*}-\bar{U}_{0}^{*}\right) z-\left(U_{2}^{*}-U_{0}^{*}\right) \bar{z}+U_{2}^{*} \bar{U}_{0}^{*}-\bar{U}_{2}^{*} U_{0}^{*}=0 .
$$

From (i), $U_{2}^{*}-U_{0}^{*}=-\rho v \mathbf{e}^{(2)}$, so the equations are $-\bar{v} \mathbf{e}^{(4)} z+v \mathbf{e}^{(2)} \bar{z}+\bar{v} V\left(s \mathbf{e}^{(5)}+r \mathbf{e}^{(2)}\right)-v \bar{V}\left(s \mathbf{e}^{(1)}+\right.$ $\left.r \mathbf{e}^{(4)}\right)=0$. The systems of equations for $\mathbf{L}_{1}, \mathbf{L}_{2}$ are

$$
\begin{gathered}
{\left[\begin{array}{ccc}
\bar{v} & -v & a(-\bar{v} V+v \bar{V}) \\
\bar{v} \omega & v & a(\bar{v} V+v \bar{V} \omega) \\
\bar{v} & v \omega & a(\bar{v} V \omega+v \bar{V})
\end{array}\right]\left[\begin{array}{c}
z \\
\bar{z} \\
1
\end{array}\right]=0,} \\
{\left[\begin{array}{ccc}
\bar{v} & v \omega & b(-\bar{v} V \omega-v \bar{V}) \\
\bar{v} & -v & b(\bar{v} V-v \bar{V}) \\
\bar{v} \omega & v & b(-\bar{v} V-v \bar{V} \omega)
\end{array}\right]\left[\begin{array}{l}
z \\
\bar{z} \\
1
\end{array}\right]=0,}
\end{gathered}
$$

where $a=s+r$ and $b=s-r$. Both determinants vanish, so $\mathbf{L}_{1}$ and $\mathbf{L}_{2}$ are each concurrent. Let $\mathbf{u}_{v}$ be the unit vector for $v$. Solving gives $K_{1}=-a \mathbf{u}_{v}^{2} \bar{V}$, $K_{2}=b \mathbf{u}_{v}^{2} \bar{V}$. So $K_{1} K_{2}=K_{2}-K_{1}=\bar{\sigma} \mathbf{u}_{v}^{2} \bar{V}$.

(iv) $U_{0}^{*}=s V \mathbf{e}^{(1)}-2 r v \mathbf{e}^{(2)}+r V \mathbf{e}^{(4)}$. Also $V+v=-3 A$ and $s-r=\frac{1}{3}$, so $U_{0_{4}}-A=\sigma v$ is parallel to $L_{4}$ through point $U_{0_{4}}$, and $A$ must lie on $L_{4}$. Similarly for $U_{0_{6}}-B=-\omega \sigma v$ and $U_{0_{2}}-C=\omega^{2} \sigma v$.

(v) A main diagonal of each generating hexagon connects a point on a vertex locus in $\mathbf{L}_{1}$ to another in $\mathbf{L}_{2}$. Thus $K_{1}$ and $K_{2}$ are not the same in general. Since the $L_{i}$ are parallel to the sides of hexagons $\boldsymbol{g}$, so must be the equilateral triangles of the sceptre.

(vi) The equations in (iii) for $K_{1}$ and $K_{2}$ show them to be collinear with the origin, and $\left|K_{1} K_{2}\right|=\bar{\sigma}|V|$, the length of the main diagonals of the identity cluster.

(vii) These special cases are readily derived from the formulas in (iii) for $K_{1}$ and $K_{2}$.

(viii) $\left|K_{1} K_{2}\right|$ is 3 times the side of $G_{0}^{*}$ since it has the same length as a main diagonal of the identity cluster. Compute $T_{1}$ and $T_{3}$, using equations in (iii) and the difference $T_{1}-T_{3}=\bar{\sigma} V$.

A sceptre is rotationally symmetric and has six equal sides and angles, so is, in a sense, a regular hexagon - albeit a disconnected one. The next theorem establishes infinite sequences of them too. Let $T^{\Delta}=\Delta_{1} \Delta_{2}=K_{1} T_{3} T_{4} K_{2} T_{6} T_{1}$ denote a sceptre, where the labeling of Lemma 7 is used for righthanded sceptres in general (see left-handed sceptre in Figure 5, right). Call the line segment $K_{1} K_{2}$ the main diagonal of a sceptre. In general, a sceptre
$T^{\Delta}$ is not centered at the origin. Let $o T^{\Delta}$ be its offset, and let $\Delta_{k} T^{\Delta}$ be its equilateral triangle $\Delta_{k}$, for $k=1$ or 2 .

We now define the sceptre constructor $\boldsymbol{\Psi}\left(H^{*}\right)$ on hexagons $H^{*}$ that are hex operator constructions on $\mathbf{P} P^{*}$. $\psi\left(H^{*}\right)$ is the corresponding dual sceptre constructor on $\mathbf{p} P^{*}$. $\Psi\left(H^{*}\right)$ is constructed, without loss of generality, as follows: (1) Form directed line segments $L_{i}$ from, say, $\left(\mathbf{i}-\mathbf{S}^{3}\right) H^{*}$ or $\left(\mathbf{i}^{2}-\varphi\right) H^{*}$. (2) If the $L_{i}$ meet the conditions defining a sceptre, return the sceptre, else $\boldsymbol{\Psi}\left(H^{*}\right)$ is undefined.

The next lemma establishes that $\boldsymbol{\Psi}\left(H^{*}\right)$ is defined for all hexagons in its domain. Let $E_{0}^{\Delta}$ be the sceptre of Lemma 7, but centered at the origin, and let $\mathbf{r}=$ $r \mathbf{u}_{v}^{2} \bar{V}$, for which $|\mathbf{r}|=0$ at $\sigma=\frac{1}{3}$. Then $T_{0}^{\Delta}=$ $\boldsymbol{\Psi}\left(\mathbf{P} P^{*}\right)=E_{0}^{\Delta}-\mathbf{r}$, so $T_{0}^{\Delta}-o T_{0}^{\Delta}=E_{0}^{\Delta}$ and $o T_{0}^{\Delta}=$ $-\mathbf{r}$. Let $0^{\Delta}$ be the sceptre of size 0 at the origin. Extend sameness and 2-3 multiples to sceptres, and extend an arbitrary hex operator $\mathbf{X}$ to sceptres by $\mathbf{X} \Psi\left(H^{*}\right)=\mathbf{\Psi}\left(\mathbf{X} H^{*}\right)$.

Lemma 8 (Sceptre operators). Let $T^{\Delta}$ be a sceptre from hex operators on $\mathbf{P} P^{*}$ of triangle $A B C$.

(i) $\mathbf{I} T^{\Delta} \cong-2 T^{\Delta}, \mathbf{B} T^{\Delta} \cong \mathbf{P} T^{\Delta}, \mathbf{p} T^{\Delta}=0^{\Delta}-2 o T^{\Delta}$, $\mathbf{i} T^{\Delta} \cong T^{\Delta}$, and $\mathbf{P}^{2} T^{\Delta}=-3\left(T^{\Delta}-o T^{\Delta}\right)+o T^{\Delta}$ are sceptres.

(ii) $\mathbf{P} T^{\Delta}$ is a sceptre with main diagonal $\sqrt{3}$ that of $T^{\Delta}$ and orthogonal to it; $\Delta_{k} \mathbf{P} T^{\Delta}$ is $\sqrt{3}$ times $\Delta_{k} T^{\Delta}$, for $k=1,2$; and $o \mathbf{P} T^{\Delta}=o T^{\Delta}$.

(iii) $\Delta_{k} T^{\Delta}$ reflected about one of its sides is coincident with $\Delta_{k} \mathbf{P} T^{\Delta}$, for $k=1,2$; that is, two vertices of $\Delta_{k} T^{\Delta}$ are collinear with two sides of $\Delta_{k} \mathbf{P} T^{\Delta}$.

Proof. The identity cluster in the general case is, without loss of generality, $W_{m}^{*}=\mathbf{i}^{m} \mathbf{I}^{a} \mathbf{P}^{b} \mathbf{i}^{c} \mathbf{p}^{d} \mathbf{B}^{e} \mathbf{P} P^{*}$. We have $W_{2}^{*}-W_{0}^{*}=(-1)^{c+d+1} 2^{b+c} \omega^{b+d+2 e} \rho v \mathbf{e}^{(2)}$, so vertex loci $L_{i}$ exist with the same orientations as in Lemma 7. The systems of equations for $\mathbf{L}_{1}$, $\mathbf{L}_{2}$ are derived and solved as there. The solution is $K_{1} K_{2}=(-1)^{c+e} 2^{a} 0^{d} \omega^{b+e}(\omega+1)^{b+e} \bar{\sigma} \mathbf{u}_{v}^{2} \bar{V}$, with midpoint at $(-1)^{a+d+1} 2^{a+d} \mathbf{r}$. This is sufficient to establish (i) and (ii). Part (iii) is proved by showing that loci $L_{i}$ and $L_{i+1}$ of $\Delta_{1} T^{\Delta}$ are concurrent with locus $L_{i}$ of $\Delta_{1} \mathbf{P} T^{\Delta}$, for $i=3,4$, which is done through calculations as in the concurrency proofs above.

So $\mathbf{B}$ is redundant here and even one application of $\mathbf{p}$ annihilates a sceptre. $\mathbf{P}$ swaps the handedness of a 
sceptre (Figure 5, right). Infinite sceptre sequences follow immediately from the lemma for any regular hexagon sequence. They can be thought of as the regular structures lying "between" the hexagons constructionally, not spatially - created by one or more identity operators $\mathbf{i}$ applied there.

Theorem 4. Each infinite regular hexagon sequence $\boldsymbol{Q}$ on truncation $P^{*}$ of triangle $A B C$ has a corresponding infinite sceptre sequence $\boldsymbol{\Psi}(\boldsymbol{Q})=\left\{\boldsymbol{\Psi}\left(Q_{j}\right): Q_{j} \in\right.$ $\boldsymbol{Q}\}$. In particular, for $j>0$ :

(i) $\boldsymbol{\Psi}(\boldsymbol{G})$ has sceptres parallel to $\boldsymbol{g}$ and to one another, strongly concentric if $\sigma=\frac{1}{3}$; diagonals collinear with one another and the centroid of $A B C$, and $\boldsymbol{\Psi}\left(G_{j}\right)$ is 2 times $\boldsymbol{\Psi}\left(G_{j-1}\right)$.

(ii) $\boldsymbol{\Psi}(\boldsymbol{H})$ has sceptres parallel to $\boldsymbol{g}$; the diagonal of $\mathbf{\Psi}\left(H_{j}\right)$ is perpendicular to that of $\mathbf{\Psi}\left(H_{j-1}\right)$ and concentric with it, and $\Delta_{k} \mathbf{\Psi}\left(H_{j}\right)$ is $\sqrt{3}$ times $\Delta_{k} \boldsymbol{\Psi}\left(H_{j-1}\right)$. (iii) $\boldsymbol{\Psi}(\boldsymbol{S})$ has sceptres parallel to $\boldsymbol{g}$ and to one another, strongly concentric if $\sigma=\frac{1}{3}$; diagonals collinear with one another and the centroid of $A B C$, and $\boldsymbol{\Psi}\left(S_{j}\right)$ is a 2-3 multiple of $\boldsymbol{\Psi}\left(G_{0}\right)$, to within translation.

(iv) $\boldsymbol{\Psi}\left(\boldsymbol{S}_{\tau}\right)$ has sceptres parallel to $\boldsymbol{g}$ and to one another, strongly concentric if $\sigma=\frac{1}{3}$; diagonals collinear with one another and the centroid of $A B C$, and $\boldsymbol{\Psi}\left(S_{\tau_{j}}\right)$ is a 2-3 multiple of $\boldsymbol{\Psi}\left(H_{1}\right)$, to within translation.

(v) $\boldsymbol{\Psi}(\boldsymbol{A})=\boldsymbol{\Psi}(\boldsymbol{S}) \cup \boldsymbol{\psi}(\boldsymbol{s}) \cup \boldsymbol{\Psi}\left(\boldsymbol{S}_{\tau}\right) \cup \psi\left(\boldsymbol{s}_{\tau}\right) \cup\left\{0^{\Delta}\right\}$ is the disjoint set of all possible sceptres from hex operators on $P^{*}$ of $A B C$, to within translation.

Figure 5 shows the first two elements of $\boldsymbol{\Psi}(\boldsymbol{G})$ (hence $\boldsymbol{\Psi}(\boldsymbol{S})$ ) and $\boldsymbol{\Psi}(\boldsymbol{H})$, two of these remarkable infinite structures, together with the corresponding hexagon sequences. The computer is invaluable for graphic study of these complex structures and how they change dynamically with $\sigma$. Small changes in $\sigma$ away

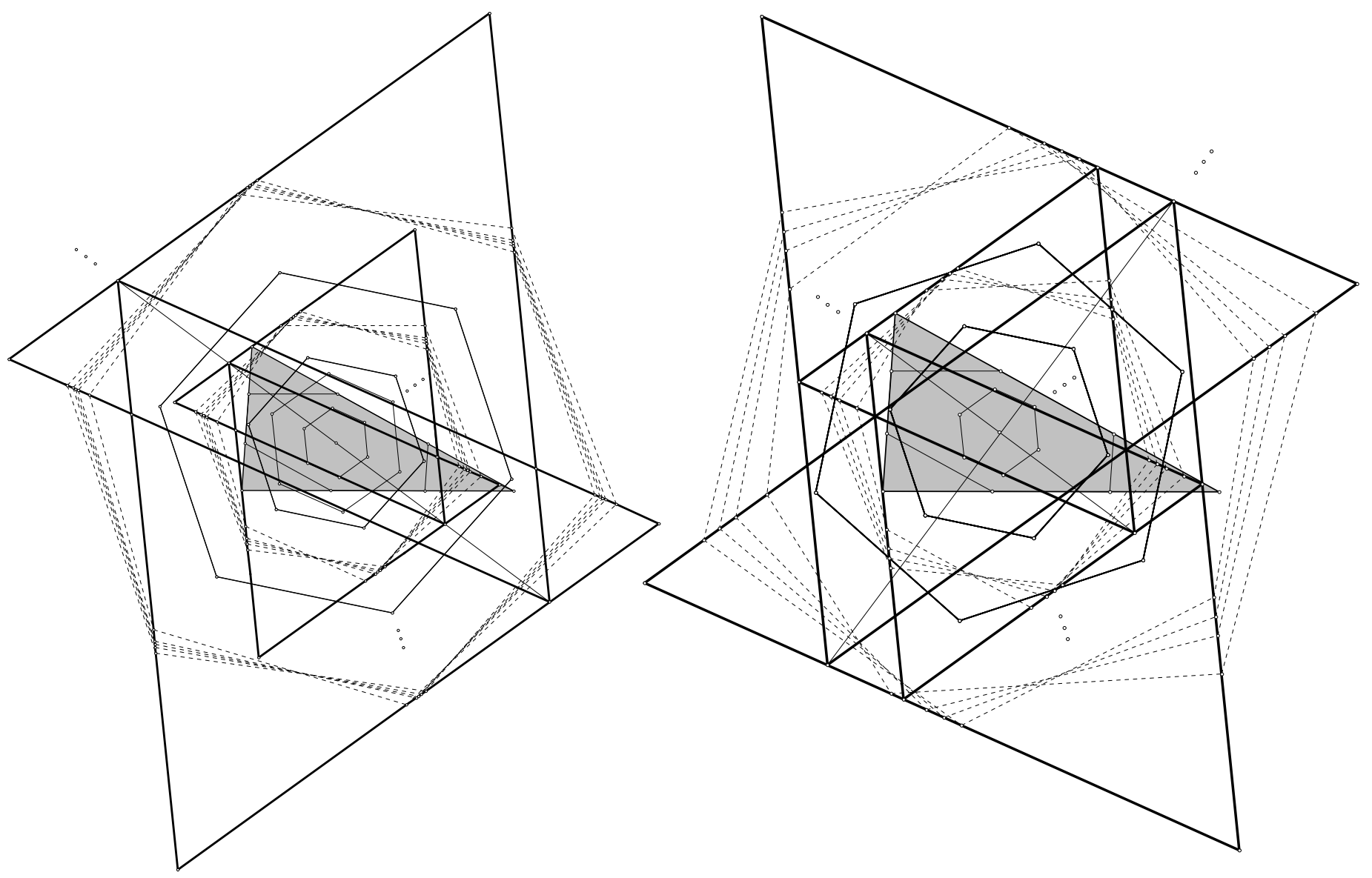

FIGURE 5. First two elements of the sequences $\boldsymbol{\Psi}(\boldsymbol{G})$ (left) and $\boldsymbol{\Psi}(\boldsymbol{H})$ (right) of sceptres (pairs of equilateral triangles) parallel to $\boldsymbol{g}$. 
from the value shown lead to complicated diagrams. The structures in the theorem hold, of course, but the generating hexagons become nonconvex and selfintersecting in irregular ways. Interaction with a diagram that varies with $\sigma$ allows one to experience the sudden appearance, near $\sigma=\frac{1}{3}$, of the configurations of Figures 4 and 5 from an apparent chaos of lines and then their abrupt disappearance back into the same complexity.

\section{REFERENCES}

[Bennett 1992] D. Bennett, Exploring geometry with the Geometer's Sketchpad, Key Curriculum Press, Berkeley, CA, 1992.

[Chang and Sederberg 1997] G. Chang and T. W. Sederberg, Over and over again, New Math. Library 39, Math. Assoc. of America, Washington, DC, 1997.

[Chapman 1997] R. Chapman, "A regular hexagon emerging from a triangle", Amer. Math. Monthly 104 (1997), 75. Solution to problem proposal 10514.
[Coxeter and Greitzer 1967] H. S. M. Coxeter and S. L. Greitzer, Geometry revisited, New Math. Library 19, Math. Assoc. of America, Washington, DC, 1967.

[Fukuta 1996a] J. Fukuta, "Problem proposal 10514", Amer. Math. Monthly 103 (1996), 267-268.

[Fukuta 1996b] J. Fukuta, "Problem proposal 1493", Math. Magazine 69 (1996), 67.

[Garfunkel and Stahl 1965] J. Garfunkel and S. Stahl, "The triangle reinvestigated", Amer. Math. Monthly 72 (1965), 12-20.

[Glassner 1999] A. Glassner, "Fourier polygons", IEEE Computer Graphics and Appl. 19 (1999), 84-91.

[Lossers 1997] O. P. Lossers, "A generalization of Napoleon's theorem", Math. Magazine 70 (1997), 7073. Solution to problem proposal 1493.

[Sketchpad n.d.] "The Geometer's Sketchpad", software, Key Curriculum Press, Berkeley, CA. See http://www. keypress.com.

[Wetzel 1992] J. E. Wetzel, "Converses of Napoleon's theorem", Amer. Math. Monthly 99 (1992), 339-351.

Alvy Ray Smith, 3420 Cascadia Avenue S, Seattle WA 98144, United States (alvyray@home.com)

Received August 4, 1999; accepted in revised form November 12, 1999 